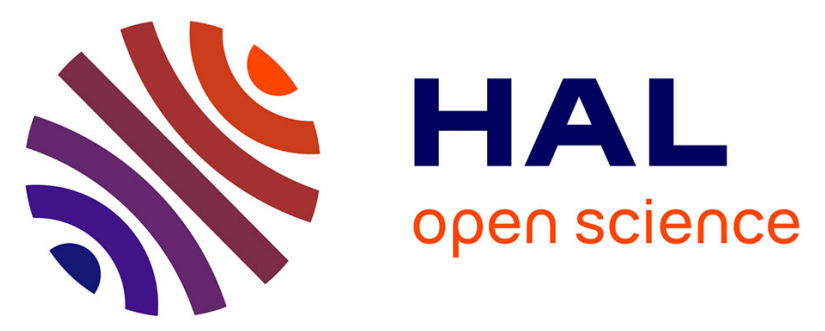

\title{
Space charge and orientational polarization within epoxy as probed by the Pulsed Electro-Acoustic technique
}

\author{
Olivier Gallot-Lavallée, G. Teyssedre, C. Laurent, S. Rowe
}

\section{To cite this version:}

Olivier Gallot-Lavallée, G. Teyssedre, C. Laurent, S. Rowe. Space charge and orientational polarization within epoxy as probed by the Pulsed Electro-Acoustic technique. Int. Conf. on Electrical Insulation and Dielectric Phenomena (CEIDP), Oct 2003, Albuquerque, United States. pp.249-252, 10.1109/CEIDP.2003.1254840 . hal-00019793v2

\section{HAL Id: hal-00019793 \\ https://hal.science/hal-00019793v2}

Submitted on 28 Feb 2006

HAL is a multi-disciplinary open access archive for the deposit and dissemination of scientific research documents, whether they are published or not. The documents may come from teaching and research institutions in France or abroad, or from public or private research centers.
L'archive ouverte pluridisciplinaire HAL, est destinée au dépôt et à la diffusion de documents scientifiques de niveau recherche, publiés ou non, émanant des établissements d'enseignement et de recherche français ou étrangers, des laboratoires publics ou privés. 


\title{
Space charge and orientational polarization within epoxy as probed by the Pulsed Electro-Acoustic technique
}

\author{
Olivier Gallot-Lavallée ${ }^{1}$, Gilbert Teyssedre ${ }^{1}$, Christian Laurent ${ }^{1}$, Stephen Rowe ${ }^{2}$ \\ ${ }^{1}$ Université Paul Sabatier, Laboratoire de Génie Electrique, Toulouse, France \\ ${ }^{2}$ Schneider Electric, Grenoble, France
}

\begin{abstract}
The Pulsed Electro-Acoustic technique, which allows probing the space and time dependence of charge density within insulators, has been applied to probe polarization and space charge phenomena within an epoxy resin under direct current stress. The response appears as a superposition of orientational polarization, due to the polar nature of epoxy resins, and space charge accumulation, both exhibiting a dynamic character considering either polarization or depolarization experiments. Attempts have been made to separate these two contributions to the space charge profile and to reconcile transient currents that can be estimated from the time dependence of space charge and those obtained from external circuit measurements. The influence of the nature of the electrodes that are used for achieving measurements on the nature of space charge is also discussed in this work.
\end{abstract}

\section{Introduction}

Electric insulations based on epoxy resin are complex materials used in electrical engineering for several years. They enter in the constitution of the supports of printed circuits, provide the insulation of certain transformers, and are more generally integrated into low and high voltage devices in which they provide mostly a double role of mechanical support and electric insulation. In spite of that, the rules of application and sizing of the resins are still empirical.

In the perspective where transport and trapping of the space charge could influence / drive ageing phenomena, our objective is to characterize space charge behaviour in an epoxy resin.

Here, we discuss the influence of the nature of the electrodes on space charge behaviour on the basis of space charge and orientational polarization analyses.

\section{Experimental protocol}

Sample: The studied samples do not contain mineral fillers, and are produced by mixing an equal weight of hardener HY 227 and resin CY 225 as referenced by Ciba Geigy. Films were processed with a thickness of about $500 \mu \mathrm{m}(+/-10 \mu \mathrm{m})$. One series of samples were not metallized, the other series was metallized using gold sputtering with electrodes of circular geometry (type 1: area $=20 \mathrm{~cm}^{2}$, thickness $=300 \AA$; type $2:$ area $=$ $0.5 \mathrm{~cm}^{2}$, thickness $=300 \AA$ ).

Space charge measurement: The electrodes of the PEA (Pulsed Electro-Acoustic) test bench are aluminium for the cathode and polymer filled with carbon black for the anode. The effective surface of probing is about $\mathrm{S}=0.5 \mathrm{~cm}^{2}$. The measurements of space charge were realized every 20 seconds under a constant DC field of $40 \mathrm{kV} / \mathrm{mm}$ applied during 1 hour, and then under short-circuit conditions of equivalent duration. The rise time of the voltage was set to $1 \mathrm{kV} / \mathrm{s}$. The amplitude of the PEA pulse was set to $300 \mathrm{~V}$, giving a pulse field of $0.6 \mathrm{kV} / \mathrm{mm}$. One measurement was performed on a metallization-free sample, the other on a metallized sample.

Current measurement: The measurements of current were realized every $2 \mathrm{~s}$ using the same protocol as for PEA measurements, on metallized samples (type 1).

\section{Results and discussions}

Space charge observation by PEA: In a general way, the definition of specific regions in space charge profiles as for example the position of the electrodes, or the limits of a charged zone, goes with an uncertainty due to signal spreading. This degradation has several origins such as the inhomogeneous acoustic dispersion in the material, the limitation in the resolution relative to the discrete acquisition $(500 \mathrm{MHz}$ rate of the oscilloscope used) and the spectral degradation during the numerical processing (relative to Gauss and Wiener filter functions) $[1,2]$. This spreading of the signal is visible on the profiles shown in Figure 1 which have been obtained on metallized and un-metallized samples at the beginning and end of the cycle of polarization / relaxation.

Space charge according to the electrode nature: Profiles shown in Figure 1.a are constituted, on the cathode size, of a polarization contribution (capacitive charge) that dominates at short polarization time and of a negative injected charge in the bulk with its corresponding image charge on the electrode, which are clearly seen at long polarization time. The influence 
charge, that is the charge on the cathode resulting from the superposition of the polarization charge and the image charge, decreases with time due to the build-up of the injected charge. The density of homocharges is important since they are visible even during the polarization. We have tentatively separated bulk and electrodes charged regions by vertical lines.

Under short-circuit conditions, the polarization charge decreases quickly since the signal in the first 20 s of depolarization appears dominated by the bulk charge (and related image). For the metallized sample, Figure $1-b$, the behaviour is quite different, since no space charge is apparent.

Space charge behaviour without metallization: In Figure 2, the whole set of profiles obtained on the metallization-free samples is shown thus allowing to follow the kinetics of charge build-up and decay. In the neighbourhood of the cathode, we can observe that the amplitude of the image charge in Volt-on decreases with time and the position of the maximum moves towards the bulk with time. In Volt-off, the polarization charge decreases quickly and the bulk charge is practically released after one hour of depolarization. Concerning what happens in the neighbourhood of the anode, the situation is not as clear as around the cathode. The most
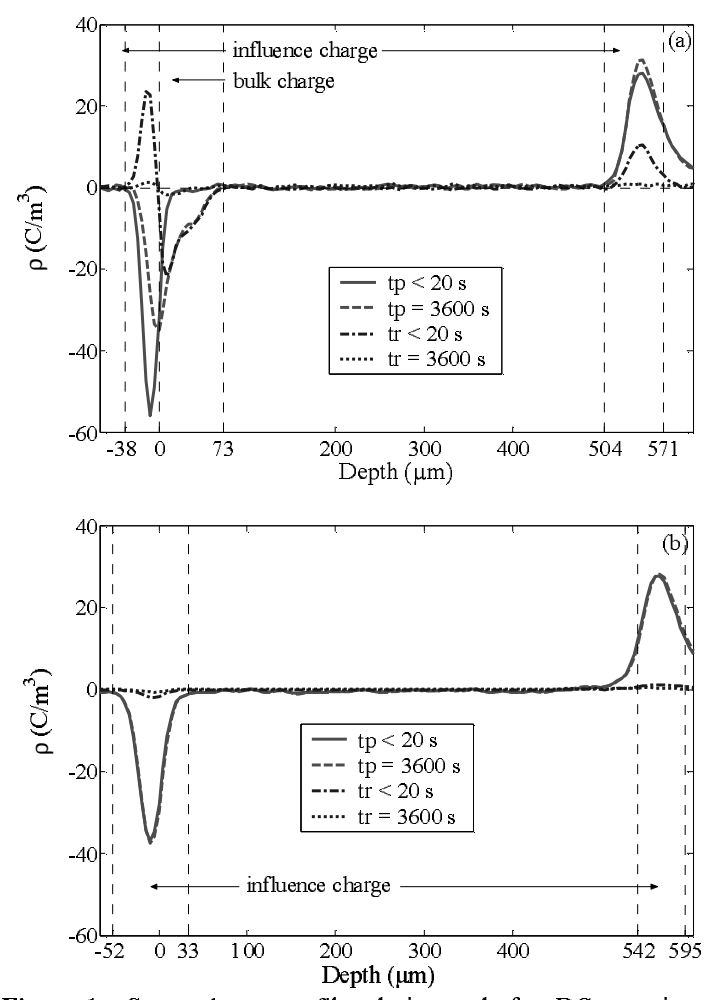

Figure 1: Space charge profiles during and after DC stressing under $40 \mathrm{kV} / \mathrm{mm}$. (a) metallization-free sample. (b) metallized sample.

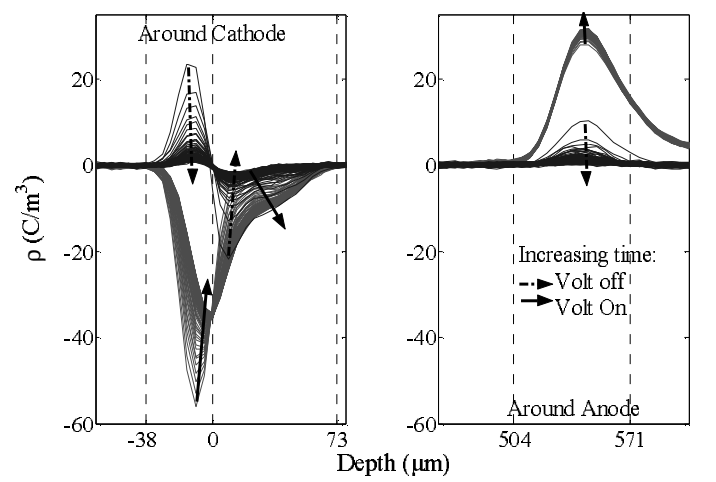

Figure 2: Space charge profiles around anode and cathode during the polarization/depolarization cycle, for an un-metallized sample.

likely scenario would be that no phenomenon of injection takes place, so that what we observe is simply the effect of a polarization of slow orientation and perhaps a weak contribution of negative homocharge. Indeed, the homocharge being much closer to the cathode than to the anode, the corresponding influence charge on the anode can be neglected (cf. Figure 3-a where a theoretical representation of the bulk and image charge is shown). Consequently, the growth of the anode charge with time would be dominated by an effect of polarization. However elements of the literature [3] as well as our own experience show that another scenario is possible, with symmetry of distribution in homocharges Figure 3-b. Thus the absence of significant signal on the anode side could be due either to a lack of injection from this electrode, or to an important degradation of the resolution that would smooth the bulk and image charge.

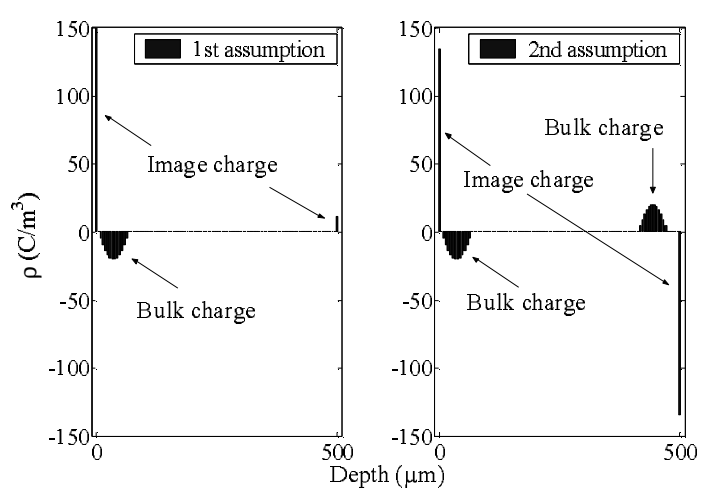

Figure 3: Left: first scenario of space charge distribution with homocharges close to the cathode. Right: second scenario with bipolar homocharges.

Analysis of charge contributions: The objective here is to separate bulk charge, influence charge and slow polarization contributions to the PEA signal. If we stay on the previous hypothesis that is to say the charge is enough confined on the respective electrodes, then we 
can write the following relation between the bulk charge and the related image charge, the index $\mathrm{K}$ referring to the cathode:

$$
Q b_{K} \approx-Q i m_{K}
$$

The influence charge, $\mathrm{Qi}_{\mathrm{K}}$, is a superposition of the image charge, $\mathrm{Qim}_{\mathrm{K}}$ and of the polarization charge $\mathrm{Qp} \mathrm{p}_{\mathrm{K}}$ :

$$
Q i_{K}=Q p_{K}+Q i m_{K}
$$

The access to the dipolar processes (slow polarization) from the space charge profiles then becomes possible considering the following relation:

$$
Q p_{K}=Q i_{K}+Q b_{K}
$$

We understand by Qim $_{K}$, the cathodic charge influenced by the associated homocharge, $\mathrm{Qb}_{\mathrm{K}}$. $\mathrm{Qp} \mathrm{p}_{\mathrm{K}}$ is used here to describe the influence charge on the cathode due to the polarization phenomenon. Finally, $\mathrm{Qi}_{\mathrm{K}}$ indicates the total charge on the cathode created by influence.

We have integrated the net charge, for each of the regions bounded by dotted lines in Figure 1, and the dynamics of build-up and relaxation of the charge is represented in Figure 4 for the cathode region of the unmetallized sample. We notice that the variations of charge are important in the first moments of the application of the stress or after the short circuit, then it becomes much slower after the first 20 minutes. This is visible in an almost symmetric way for the influence charge and the bulk charge in Figure 4, thus revealing that the variation in influence charge is primarily due to the build-up of the injected charge (weak contribution of delayed polarization).

In Figure 5, we have represented the polarization contribution for each sample on each side. In the case of metallization-free sample and on the cathode side, $Q p_{K}$ was obtained according to the relation (3); in the other cases it is assumed that bulk charge effect is insignificant because it is either far away from anode, or null. We observe that the polarization increases during the time under stress, which appears consistent with a phenomenon of slow polarization supposed present in this kind of material. We also observe that the polarization of the un-metallized sample, Figure 5-a, seems to be slightly more important than for the metallized one, Figure 5-b. However, this observation is highly dependent on the limits of integration that are taken in an almost arbitrary way. The other noticeable behaviour is that the amount of delayed polarization appears lower for the metallized sample.

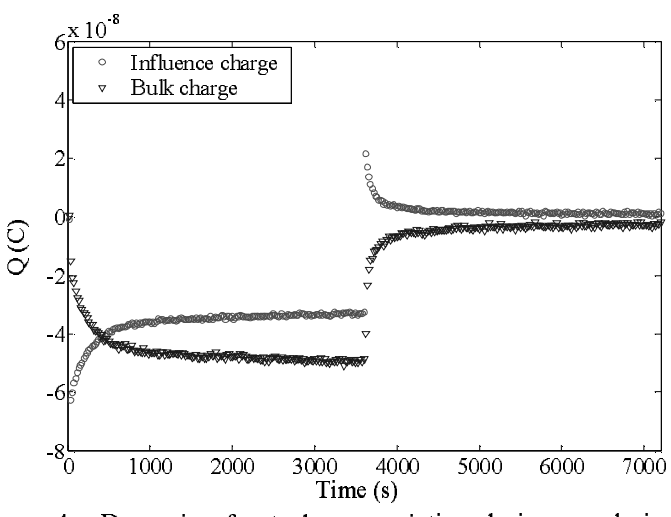

Figure 4: Dynamic of net charge variation during a polarisation / relaxation cycle at the cathode of a metallization-free sample. Quantities were integrated in the regions limited by dashed lines in Figure 1 and are relevant to $0.5 \mathrm{~cm}^{2}$ area of the sample.

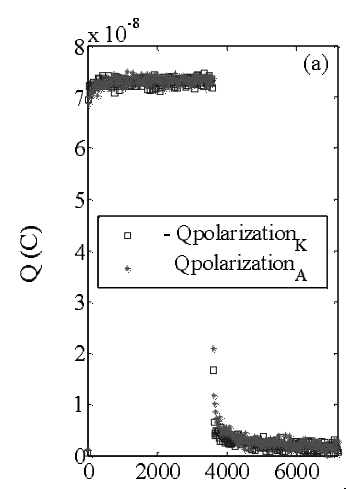

Figure 5: Dynamic of the polarization charge during a polarisation / relaxation cycle. (a) metallization-free sample; (b) metallized sample. Quantities are relevant to $0.5 \mathrm{~cm}^{2}$ area of the sample.

Analysis of current contributions: In the following, we attempt to transpose the results of space charge in terms of current variations. Supposing that internal generation of charge, through e.g. field-induced dissociation processes, is negligible, then injection and polarization remain the two contributing phenomena to space charge behaviour. In this case, the equivalent currents deduced from space charge profiles can be connected in theory by the following relation (4):

$$
-d / d t\left(Q p_{K}+Q b_{K}\right)=I e x t-I c o n d
$$

where Iext is the external current and Icond is the steady state conduction current $\left(0.13 \mathrm{pA} / \mathrm{cm}^{2}\right.$ as obtained from the difference between experimental polarization and relaxation currents). We have represented in Figure 6 the currents deduced from space charge measurement on the metallized sample. Note that in that case, Qbk is considered as null, and hence the polarization current is to be compared to the external current. 


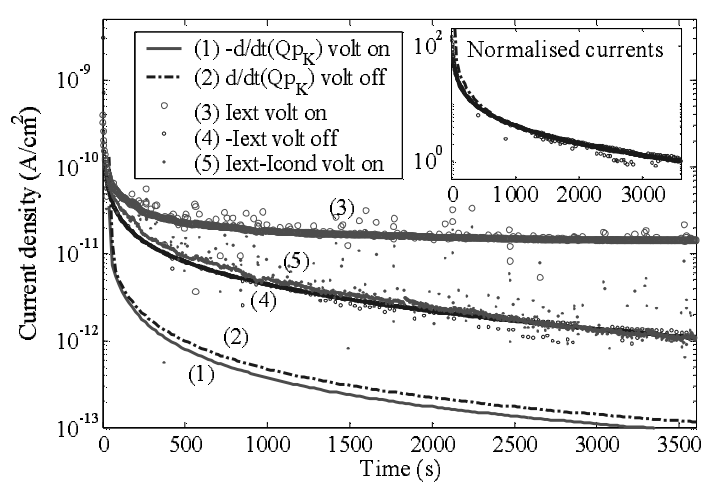

Figure 6: External currents measured and external currents deduced from space charge measurement on a metallized sample. In inset, normalized currents (2) and (4) are compared.

These 'PEA currents' have been obtained by fitting charge curves as shown in Figures 4 and 5 and then by derivation of the obtained function. We observe that the 'PEA' currents obtained in Volt-on and Volt-off are very similar, and it is true also for the experimental currents, once the conduction contribution has been removed. There is however a significant quantitative difference (around 1 decade) between 'PEA' and experimental currents. This quantitative difference has not been completely understood. We have notably observed a stronger experimental current for measurements achieved in air (data shown here) compared to vacuum conditions [4]. Despite the quantitative problem to be fixed, on a qualitative aspect, experimental and 'PEA' currents exhibit the same behaviour as shown in the inset of Figure 6 considering normalized values of the current.

Figure 7 compares 'PEA' external currents for metallized and un-metallized samples. The current for the latter is much higher than for the former. This behaviour appears consistent with the fact that without metallization significant charge injection occurs; the related current dominates largely over delayed polarization. It seems therefore that extraction of current from PEA measurements is achievable, with as attractive feature the possibility to separate different current sources.

\section{Conclusions}

All considered two informations emerge: on a material point-of-view, evidence of space charge has been given for a non-metallized sample, whereas it did not appear on a metallized sample in our experimental conditions; on a technologic point-of-view the PEA technique seems to be a good tool to extract different contributions to the current even if there are still question marks about quantitative information.
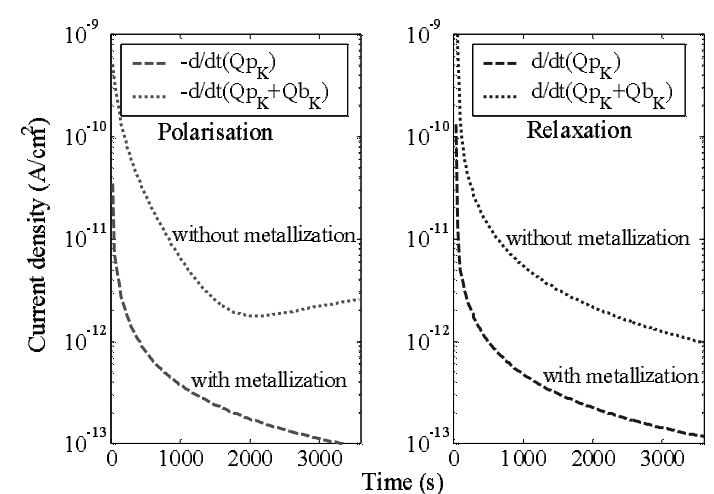

Figure 7: External currents deduced from space charge measurement on a metallization-free sample and a metallized sample. Left: volt-on; Right: volt-off.

Regarding the enhancement of injection in nonmetallized samples, there are two possible reasons: either gold acts as an efficient barrier to injection as compared to a semiconducting electrode, or the silicon oil used for mechanical coupling between the sample and the electrode has some role.

Regarding extraction of currents from PEA, this requires derivation of the net charge measured, which has been done in the present case considering that the charge varies in time according to a superposition of exponential functions. The quality of the result therefore depends on the adequacy of the model to phenomena at play and on the quality of the fit. Note finally than quantitative discrepancies which have been observed appear more in relation with conditions in which the external current has been measured and its dependence with ambient atmosphere (vacuum, humidity...) than on the mathematical method used for extracting current from space charge data.

\section{References}

[1] O. Gallot-lavallée, "Space charge measurement in solid dielectrics by the pulsed electro-acoustic technique", submitted to Eur. J. Phys.-A.P.

[2] M. Jeroense, "Charges and discharges in HVDC Cables", Ed. Delft University Press, Delft, The Netherlands, p. 185, 1997.

[3] V. Griseri, "The effect of high electric fields on an epoxy resin", $\mathrm{Ph}$. D. Thesis, Engineering Dept., Univ. of Leicester UK, p. 114, 2000.

[4] O. Gallot-lavallée, "Polarisation et charge d'espace dans une resine époxy par mesure Electro Acoustique Pulsée", Actes du Colloque sur les Matériaux du Génie Electrique, pp. 143-145, Grenoble, France, Avril 2003.

Author address: Olivier Gallot-lavallée, Laboratoire de Génie Electrique de Toulouse, 118 route de Narbonne, 31062 Toulouse, France, Email:gallot-lavallee@1get.ups-tlse.fr 